ORIGINAL ARTICLE

\title{
Chest physiotherapy, gastro-oesophageal reflux, and arousal in infants with cystic fibrosis
}

\author{
B M Button, R G Heine, A G Catto-Smith, P D Phelan, A Olinsky
}

Arch Dis Child 2004;89:435-439. doi: 10.1136/adc.2003.033100

See end of article for authors' affiliations .....................

Correspondence to: Dr A G Catto-Smith, Dept of Gastroenterology and Clinical Nutrition, The Royal Children's Hospital Parkville, Melbourne, Victoria 3052, Australia; tony.cattosmith@ rch.org.au

Accepted 16 September 2003
Background: Postural drainage chest physiotherapy in infants with cystic fibrosis (CF) exacerbates gastrooesophageal reflux (GOR) and may contribute to a more rapid deterioration in lung function.

Aims: To compare standard postural drainage chest physiotherapy (SPT) and a modified physiotherapy regimen (MPT) without head-down tilt, with regard to GOR, arousal state, and cardiorespiratory function. Methods: Twenty infants with CF underwent 30 hour oesophageal pH monitoring, during which four chest physiotherapy sessions were administered (day 1: MPT-SPT; day 2: SPT-MPT). Arousal state, heart rate, and oxygen saturation were documented for each of the physiotherapy positions (supine, prone, right lateral, and left lateral with (SPT) or without (MPT) $30^{\circ}$ head-down tilt).

Results: Significantly more reflux episodes occurred during SPT than during MPT, but there were no significant differences in median episode duration or fractional reflux time. During SPT, left lateral positioning was associated with fewer reflux episodes compared to other positions. During supine and prone positioning, more reflux episodes occurred during SPT than during MPT. Infants were significantly more likely to be awake or cry during SPT. There was a significant association between crying and reflux episodes for SPT. Non-nutritive sucking was associated with a significant reduction in reflux episodes during SPT. Oxygen saturation during SPT was significantly lower during crying and other waking, and non-nutritive sucking during SPT was associated with a significant increase in oxygen saturation. Conclusions: SPT is associated with GOR, distressed behaviour, and lower oxygen saturation.
G astro-oesophageal reflux (GOR) occurs frequently in infants with cystic fibrosis (CF). ${ }^{12}$ The detection of $\mathrm{CF}$ by screening methods carried out in all neonates has led to the earlier use of chest physiotherapy in this presymptomatic population. Previous studies carried out by our group have shown that in some children, head-down postural drainage chest physiotherapy exacerbates $\mathrm{GOR}^{3}$ and may contribute to a more rapid deterioration in lung function ${ }^{2-5}$ compared to a modified regimen without head-down tilt. This has led to a change in the recommendations for standard chest physiotherapy in infants with CF. ${ }^{6}$

Several factors have an impact on GOR. Reflux episodes in infants and adults occur most commonly during transient lower oesophageal sphincter relaxations. ${ }^{78}$ The frequency of these events is influenced by sucking, swallowing, gastric distension, dietary fat content, arousal state, ${ }^{10}$ age, and body positioning. ${ }^{11}$ It is not clear what role, if any, these factors have in contributing to clinically significant GOR and cardiorespiratory disturbances.

The aims of our study were to examine the effects of two different chest physiotherapy regimens on GOR, arousal state, and cardiorespiratory function in young infants with CF.

\section{PATIENTS AND METHODS}

We invited participation from the parents of all infants newly diagnosed with CF between February 1993 and December 1994. Approval for the study had been given by the Ethics in Human Research Committee of the Royal Children's Hospital, and informed written consent was obtained from all participating parents.

\section{Oesophageal pH monitoring}

After the diagnosis of $\mathrm{CF}$ and parental counselling, infants underwent 30 hour oesophageal $\mathrm{pH}$ monitoring using an ambulatory system (Digitrapper, Synectics, Sweden) with antimony $\mathrm{pH}$ electrodes, as previously described. ${ }^{3}$ The $\mathrm{pH}$ probe was introduced through the nose and the tip sited at $87 \%$ of the distance from the nares to the lower oesophageal sphincter using a nomogram based on body length. ${ }^{12}$ The position of the probe was confirmed by a change in acidity on withdrawal from the stomach, and radiology obtained if necessary to confirm tube position. A reference electrode was attached to the anterior chest wall. The $\mathrm{pH}$ probe and reference electrodes were connected to a $\mathrm{pH}$ recording device which included an event marker and clock. A detailed diary was kept recording the timing and duration of feeds, crying, body posture, sleep, and physiotherapy. At the end of the recording period the data were downloaded for analysis.

Reflux parameters were analysed for the period of the 30 hour study. The number and duration of reflux episodes with $\mathrm{pH}$ less than 4.0 and lasting longer than 15 seconds were recorded. The frequency of reflux episodes was expressed as episodes per hour, which in the case of reflux during chest physiotherapy involved pro rata multiplication of the number of reflux episodes as the duration of physiotherapy was less than one hour. Fractional reflux time (FRT), which represents the proportion of the total time of the recording for which the oesophageal $\mathrm{pH}$ was less than 4.0 was calculated and represented as a percentage.

Chest physiotherapy was undertaken under one of two regimens: "standard" (SPT), including postural drainage positions, or "modified" (MPT), without head-down tilt. ${ }^{3}$ The number of reflux episodes per hour, duration of reflux episodes, and FRT were calculated for each of the positions during SPT and MPT.

Abbreviations: CF, cystic fibrosis; $\mathrm{Cl}$, confidence interval; FRT, fractional reflux time; GOR, gastro-oesophageal reflux; MPT, modified chest physiotherapy; NNS, non-nutritive sucking; SPT, standard chest physiotherapy 


\section{Chest physiotherapy}

Chest physiotherapy sessions were carried out at least two hours after feeds. During the 30 hour period of oesophageal $\mathrm{pH}$ monitoring, each patient received four sessions of chest physiotherapy. These consisted of two sessions of standard physiotherapy (SPT: supine horizontal, prone $30^{\circ}$ head-down, right lateral $30^{\circ}$ head-down, and left lateral with $30^{\circ}$ headdown) and two sessions of modified physiotherapy (MPT: supine $30^{\circ}$ head-up, prone horizontal, right lateral horizontal, and left lateral horizontal). ${ }^{3}$

In each position, four cycles of two minutes' duration of percussion, followed by six vibrations (thoracic compressions) were carried out. The infants remained in the position for a short rest during which oxygen saturation and heart rate were measured and the patients' state of arousal was recorded. On day 1, MPT was undertaken in the morning, and SPT in the afternoon. The order was reversed on day 2 . The order of the individual positions during MPT or SPT sessions was randomised.
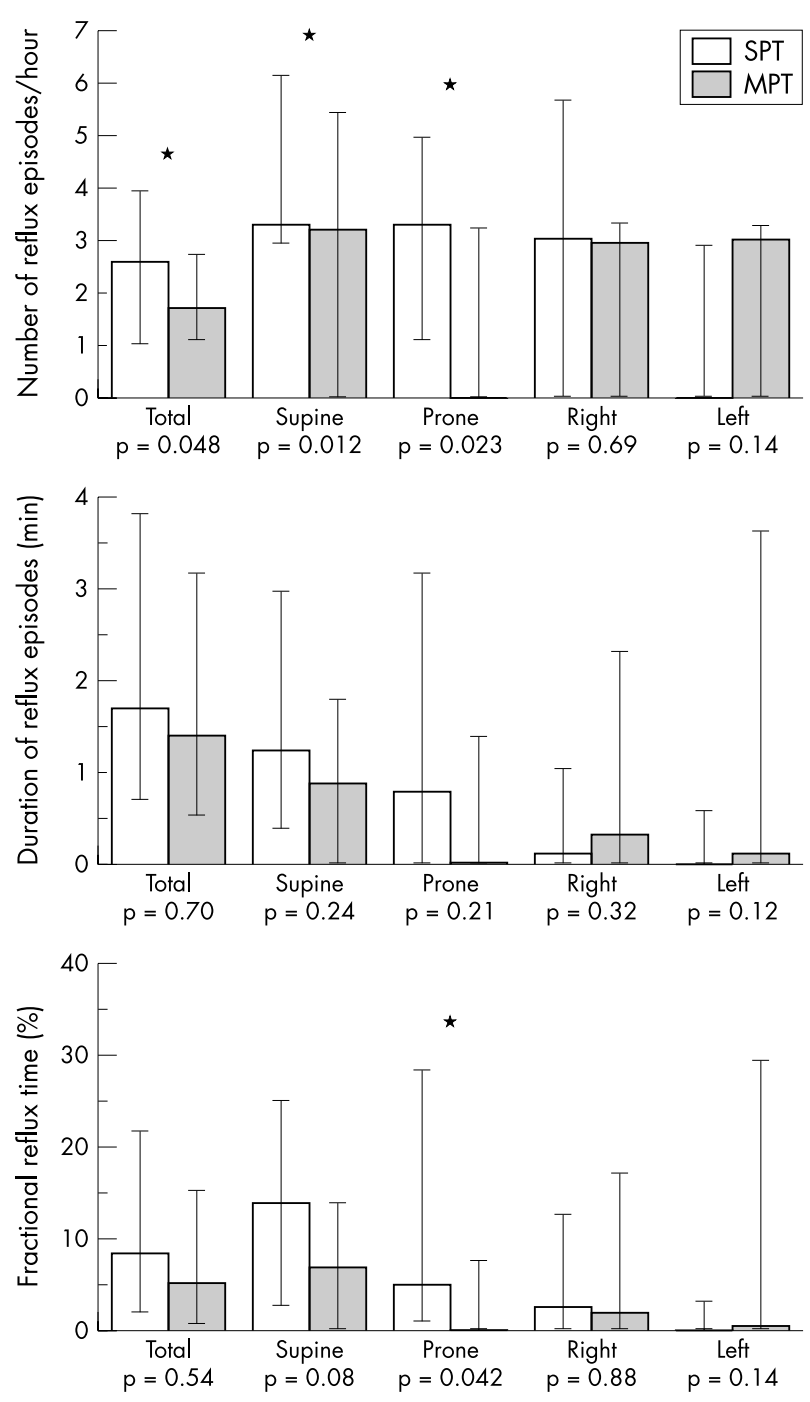

Figure 1 Median number of reflux episodes per hour, median duration of reflux episodes, and median fractional reflux time during chest physiotherapy in each of four positions carried out using either a standard postural drainage technique (SPT), or a modified regimen without head-down tilt (MPT). Twenty patients were studied twice in each of four positions during both physiotherapy regimens. Error bars show interquartile range. ${ }^{*}$ Wilcoxon signed rank test: $p<0.05$.
Heart rate, oxygen saturation, and arousal state during chest physiotherapy

Oxygen saturation and heart rate were recorded using a Nellcor Symphony N-3000 Pulse Oximeter (Nellcor; Pleasanton, CA). The oximetry probe was placed over the end of the hallux. Oxygen saturations and heart rate were recorded simultaneously. Movement artefact was carefully avoided by taking all measurements during rest periods after a clear pulsatile trace had been established. Baseline resting measurements of oxygen saturation and heart rate were recorded prior to each physiotherapy session.

Oxygen saturation, heart rate, and state of arousal were recorded during a two minute rest period following the eight minute cycle in each position. The arousal state was divided into five different categories: quiet sleep, sleep while sucking on a pacifier, quiet wakefulness, wakefulness while sucking on a pacifier, and crying. Infants used to pacifiers were comforted with one if necessary. Where arousal status changed during a treatment position, the predominant state was recorded.

\section{Statistical analysis}

Data are presented as mean (SD) for normally distributed data, and as the median and interquartile range for skewed distributions. Normally distributed data were compared using Student's $t$ test. The Wilcoxon signed rank test was used for paired non-parametric comparisons between physiotherapy regimens. Analysis of variance (ANOVA) was used for multiple comparisons of continuous variables within physiotherapy regimens. $\chi^{2}$ analysis was performed to test for associations between categorical data. Statistical significance was accepted for $\mathrm{p}<0.05$.

\section{RESULTS \\ Population}

During the study period, 27 infants were diagnosed with CF, and the parents of 20 consented to participate in the study. The mean age of these 20 infants at the time of enrolment was 2.1 months (range $1-4$ months). Sixteen infants had been detected through neonatal screening by blood immunoreactive trypsinogen, and four infants had presented with meconium ileus. The diagnosis of CF was confirmed by mutation analysis in infants who were homozygous for the $\Delta$ F508 mutation, or by pilocarpine sweat iontophoresis (sweat chloride $>60 \mathrm{mmol} / \mathrm{l}$ ) in infants with rare mutations or who were compound heterozygous for $\Delta \mathrm{F} 508$.

\section{GOR during chest physiotherapy (fig 1)}

A total of 160 SPT positions and 160 MPT positions were available for analysis. Overall, there were significantly more reflux episodes during SPT compared to MPT ( $p<0.05$ ). The duration of reflux episodes and FRT was similar during SPT and MPT.

We examined the relation between individual physiotherapy positions and GOR. During SPT, there were significantly fewer episodes of reflux per hour while placed in the left lateral position compared to supine, prone, or right sided positioning (ANOVA, $\mathrm{p}=0.007$ ). There were no significant differences in the duration of reflux episodes $(p=0.09)$ or in FRT between individual positions during SPT $(p=0.12)$. During MPT there were no significant differences in the number of reflux episodes $(p=0.22)$, their duration $(p=0.575)$, or in FRT $(p=0.75)$ between any of the positions.

We then compared the effect of the four different physiotherapy positions on GOR for the two physiotherapy regimens. There were significantly more reflux episodes during supine and prone SPT positions than during MPT. The duration of reflux episodes failed to reach a significant 
difference between regimens during supine (SPT 2.26 (2.97) $v$ MPT 1.195 (1.32), $\mathrm{p}=0.096$ ) or prone positioning (SPT 1.77 (2.29) $v 1.16(2.00), \mathrm{p}=0.089)$.

\section{Arousal states, GOR, and chest physiotherapy (table 1)}

Comparing physiotherapy regimens, infants were significantly more likely to be awake $\left(\chi^{2}=3.87, \mathrm{p}=0.048\right)$ or crying during SPT than during MPT $\left(\chi^{2}=10.99, \mathrm{p}=0.0009\right)$. There were no other significant differences in the frequency of arousal states between the two physiotherapy regimens once crying was excluded from analysis.

During SPT, reflux episodes were significantly less frequent when asleep $(p=0.033)$. During MPT, there was no significant difference in the frequency of reflux episodes in relation to arousal state $(p=0.253)$. When we compared SPT and MPT regimens, reflux episodes were more likely to occur during SPT when the infant was crying or upset than during $\operatorname{MPT}(\mathrm{p}=0.02)$.

Non-nutritive sucking was associated with a significant reduction in reflux episodes during SPT but not during MPT (SPT: $\chi^{2}=6.07, \mathrm{p}=0.01 ;$ MPT: $\chi^{2}=0.38, \mathrm{p}=0.54$ ).

\section{Impact of GOR on heart rate and oxygen saturation}

During SPT there were 156 positions available for analysis. Of these, 52 had at least one episode of reflux. Comparing physiotherapy positions during which reflux occurred $(\mathrm{n}=52)$ with non-reflux positions $(\mathrm{n}=104)$, there were no significant differences in heart rate (reflux positions 145 (15.8) $v$ non-reflux positions 140 (16.4) per minute; $\mathrm{p}=0.07$ ) or oxygen saturation (reflux positions $98.5(2.52) \% v$ nonreflux positions $98.6(1.91) \%$; $\mathrm{p}=0.904)$.

During MPT, GOR occurred during 41 of 160 positions. There were no significant differences in heart rate or oxygen saturation between positions in which reflux occurred $(n=41)$ versus non-reflux positions $(n=119)$ : heart rate during reflux positions 135 (22.9) per min $v$ non-reflux positions 141 (14.3) $(\mathrm{p}=0.125)$; and oxygen saturation during reflux positions 98.7 (1.77)\% $v$ non-reflux positions $98.8(1.56) \%(\mathrm{p}=0.69)$.

\section{Impact of arousal state on heart rate and oxygen saturation (table 2)}

During SPT, heart rates were significantly higher during crying than during other states (crying heart rate 153.4 (17.1) per $\min (\mathrm{n}=41) \quad v \quad 137.5 \quad(13.9) \quad(\mathrm{n}=115)$ per min; $\mathrm{p}<0.001)$. Oxygen saturation was significantly lower during crying than in other states $(97.6(3.17) \%(\mathrm{n}=41) \vee 98.9$ $(1.49) \%(n=115) ; p=0.02)$. Heart rate was significantly lower when asleep, even when crying was excluded from the "awake" analysis (asleep 132.0 (13.0) per min $(\mathrm{n}=52) v$ awake 142.1 (13.0) per min $(\mathrm{n}=63)$; $\mathrm{p}<0.001)$. Oxygen saturation was significantly higher when asleep during SPT, even when crying was excluded (asleep 99.2 (1.15)\% $(\mathrm{n}=52)$ $v$ awake $98.5(1.66) \%(\mathrm{n}=63) ; \mathrm{p}=0.01)$.

Pacifiers were used during both physiotherapy regimens in similar frequencies $(p=0.69)$. Non-nutritive sucking with a pacifier during SPT was associated with a significantly lower heart rate, even after excluding crying (non-nutritive sucking 135.4 (13.1) per min $v$ not sucking 141.4 (14.4) per min; $\mathrm{p}=0.031)$. Oxygen saturation during pacifier use was significantly higher (non-nutritive sucking 99.1 (1.14)\% v not sucking $98.4(1.9) \% ; \mathrm{p}=0.031)$.

During MPT, crying resulted in a significant increase in heart rate but had no effect on oxygen saturation (crying heart rate 156.3 (14.4) per min $(\mathrm{n}=18) v$ not crying 137.0 (16.1) per min $(\mathrm{n}=142) ; \mathrm{p}<0.001)$. Heart rate was significantly lower in MPT when asleep compared to wakeful periods, even when crying was excluded from analysis (asleep 134.0 (11.1) per min $(\mathrm{n}=68) v$ awake 143.0 (19.5) per min $(\mathrm{n}=92) ; \mathrm{p}<0.001)$. Oxygen saturation was similar during sleep and wakeful periods in MPT (awake 98.7 $(1.44) \%(\mathrm{n}=74) v$ asleep $98.9(1.44) \%(\mathrm{n}=68) ; \mathrm{p}=0.389)$. Non-nutritive sucking was not associated with any significant difference in heart rate $(p=0.573)$ or oxygen saturation $(\mathrm{p}=0.515)$.

\section{DISCUSSION}

In general, infants appeared to tolerate both chest physiotherapy regimens during our study well and displayed crying or distressed behaviour for less than $20 \%$ of the total physiotherapy periods. Some differences emerged between the two physiotherapy regimens. Children undergoing MPT were more likely to sleep during the procedure than those undergoing SPT. SPT, in turn, was associated with increased distressed behaviour compared to MPT. There were more episodes of GOR during SPT. This increase in reflux episodes during SPT was particularly marked during supine and prone positioning, compared to MPT. The left lateral position appeared to be partially protective against reflux, but only in SPT. GOR episodes were more frequent during crying in SPT, but reduced during sleep and non-nutritive sucking.

In the present study, SPT was associated with an increased number of reflux episodes, compared to MPT. However, there was no statistical difference for the FRT, except in the prone position. Furthermore, the median duration of reflux episodes, a marker of oesophageal acid clearance, was similar for both regimens. The FRT is mainly used as a measure of distal oesophageal acidification which relates to the risk of acid-peptic mucosal injury-that is, reflux oesophagitis. It

\begin{tabular}{|c|c|c|c|c|c|}
\hline Arousal state & $\begin{array}{l}\text { Total } \\
\text { n (\%) }\end{array}$ & $\begin{array}{l}\text { MPT } \\
\text { n (\%) }\end{array}$ & $\begin{array}{l}\text { SPT } \\
\text { n (\%) }\end{array}$ & $\chi^{2}$ & p value \\
\hline 1: Asleep & $43(13.4)$ & $28(17.5)$ & $15(9.4)$ & & \\
\hline RE & & & & 1.41 & 0.23 \\
\hline 2: Asleep with NNS & $75(23.4)$ & $40(25.0)$ & 35 (21.9) & & \\
\hline $\mathrm{RE}$ & 12 & 7 & & 0.14 & 0.7 \\
\hline 3: Awake and settled & $76(23.8)$ & $45(28.1)$ & $31(19.3)$ & & \\
\hline RE & 30 & 15 & 15 & 1.74 & 0.18 \\
\hline 4: Awake with NNS & $67(20.9)$ & $29(18.1)$ & $38(23.8)$ & & \\
\hline RE & 23 & 9 & 14 & 0.25 & 0.62 \\
\hline 5: Crying/upset & $59(18.4)$ & $18(11.3)$ & $41(25.6)$ & & \\
\hline$R E$ & 19 & 2 & 17 & 5.28 & 0.02 \\
\hline \multicolumn{6}{|c|}{$\begin{array}{l}\text { There were } 160 \text { positions available for analysis during each of the MPT and SPT regimens. } \\
\mathrm{n} \text {, number of positions with that arousal state }(\% \text { of } 160) \text {. } \\
\text { RE, number of positions with at least one reflux episode. } \\
\chi^{2} \text { compares reflux episodes for each arousal state between physiotherapy regimens. }\end{array}$} \\
\hline
\end{tabular}


Table 2 Oxygen saturation during individual arousal states in MPT and SPT

\begin{tabular}{|c|c|c|c|c|c|c|c|}
\hline \multirow[b]{2}{*}{ Arousal state } & \multicolumn{3}{|c|}{ MPT } & \multicolumn{3}{|c|}{ SPT } & \multirow[b]{2}{*}{ p value } \\
\hline & $n$ & Mean $(\%)$ & (SD) & $n$ & Mean $(\%)$ & (SD) & \\
\hline 1: Asleep & 28 & 98.7 & (1.67) & 16 & 99.7 & $(0.60)$ & 0.009 \\
\hline 2: Asleep with NNS & 40 & 99.1 & (1.26) & 36 & 99.0 & (1.28) & 0.94 \\
\hline 3: Awake and settled & 45 & 98.7 & $(1.36)$ & 25 & 97.6 & $(1.98)$ & 0.012 \\
\hline 4: Awake with NNS & 29 & 98.7 & (1.59) & 38 & 99.2 & (1.01) & 0.12 \\
\hline 5: Crying/upset & 18 & 98.3 & (2.64) & 41 & 97.6 & (3.17) & 0.38 \\
\hline
\end{tabular}

Data are expressed as percentage oxygen saturation.

$\mathrm{n}$, number of positions during which this was the predominant arousal state.

correlates poorly with the respiratory manifestations of GOR as oesophageal pH monitoring cannot determine whether refluxed gastric contents reach the airway or not-even when using upper oesophageal or pharyngeal $\mathrm{pH}$ probes. It appears plausible that the risk of aspiration increases with the number of reflux episodes. We therefore speculate that the number of reflux episodes may be a more meaningful parameter than the FRT in assessing the risk of reflux associated pulmonary complications.

Previous workers have examined the effect of positioning on reflux. Orenstein and colleagues ${ }^{13}$ identified a reduced FRT in the twohour post-prandial period during horizontal and head-up prone positioning, compared with supine seated. A subsequent study identified a marginal benefit to the head-upright prone position compared to prone horizontal in the frequency of post-prandial reflux episodes and the frequency of post-prandial reflux episodes longer than five minutes. ${ }^{14}$ There was no significant difference in reflux parameters between head-elevated prone and flat prone during the "fasting" period greater than two hours after feeds.

Our studies were carried out in prone, supine, and left and right lateral positions, more than two hours after a feed. We examined reflux parameters during chest physiotherapy in this period. It is important to recognise that the positions we used were not identical to those used by Orenstein. More importantly, our study was carried out with simultaneous chest physiotherapy. We found significantly more reflux episodes during chest physiotherapy undertaken in the $30^{\circ}$ head-down prone and supine positions compared to their head-up equivalents. Importantly, this occurred during the "fasting" period, during which the unbuffered gastric contents provide little protection against acid-peptic injury. Prone positioning has been suggested as being protective against reflux, ${ }^{15}$ but its therapeutic value has been questioned $^{14}$ when studied in the head-up or flat positions. The mechanism for the increase in reflux episodes during headdown prone positioning may relate to increased intraabdominal and gastric fundal pressure, and more frequent lower oesophageal sphincter relaxations. ${ }^{16}$ The left lateral position has been identified as "protective" in children with otherwise uncomplicated reflux..$^{15}$ In our studies, the left lateral position was protective only during SPT. We speculate that this effect may in part be because the lower oesophageal sphincter falls below the gastric "air-fluid level" when in the left decubitus position. ${ }^{13}$

Reflux was significantly reduced in sleep and during nonnutritive sucking, although this was confined to SPT. The reduction in GOR that we observed during sleep is well recognised, but appears to be more marked in patients with significant GOR compared to non-reflux patients. ${ }^{10}$ In our study, SPT was associated with significantly more reflux than MPT. We found no evidence of any significant effect of arousal state on reflux in MPT positions. The effect of nonnutritive sucking on GOR has been the subject of relatively few studies, and the outcomes of these are confusing.
Orenstein 9 found that pacifier use in infants with pathological reflux was associated with a reduction in reflux episodes if infants were seated upright, but an increase in GOR during the post-prandial period in prone positioned infants. In our study, non-nutritive sucking during SPT appeared to have a protective effect on GOR. Several differences exist between our study population and that of Orenstein. Our patients had $\mathrm{CF}$, underwent chest physiotherapy, and were not studied in the same body positions. Furthermore, the study lacked statistical power to discriminate any significant differences in GOR parameters for each of the SPT positions in relation to pacifier use.

Crying was associated with an increase in reflux in SPT positions in our patients. Furthermore, infants undergoing SPT cried more often. We were unable to determine whether these infants cried because of reflux induced "heartburn", or whether crying induced GOR. Other studies have identified a link between crying and reflux. ${ }^{17}$ However, at least one previous study in otherwise normal infants produced the surprising finding that crying did not increase reflux, ${ }^{18}$ and others have failed to establish a temporal relation between crying and reflux episodes. ${ }^{19}{ }^{20}$ Our population was different to those previously reported; we may have precipitated reflux episodes through postural drainage chest physiotherapy, while other studies of the association of reflux with crying were observational.

Compared to sleep, the heart rate during crying and wakefulness were increased in both SPT and MPT, and oxygen saturation was reduced in these states in SPT only. Additionally, non-nutritive sucking was associated with a reduction in the frequency of reflux episodes as well as a lower heart rate, but improved oxygen saturation. This effect was limited to SPT. Within our study population, we found no evidence of a significant change in heart rate or oxygen saturation in relation to reflux episodes. Not unexpectedly, we found an increase in heart rate during crying during both SPT and MPT, but only in SPT was there an associated reduction in oxygen saturation. Other studies have shown that oxygen saturation is reduced in sick patients tilted headdown for physiotherapy, ${ }^{21-23}$ although the effect of crying has not been examined specifically. Again, not unexpectedly, heart rate was less during sleep in both SPT and MPT. There was, however, an increase in oxygen saturation during sleep in SPT.

Non-nutritive sucking during SPT was associated with a reduction in heart rate and increased oxygen saturation. No significant effect was noted during MPT. There is a large volume of literature on the effects of non-nutritive sucking on heart rate and oxygenation, ${ }^{24-28}$ but no previous studies have examined the effect of non-nutritive sucking on GOR during chest physiotherapy.

In summary, we have identified numerous differences between MPT and SPT in terms of effects on GOR, arousal, and cardiorespiratory function. Our previous studies on the effects of SPT in children with CF have highlighted the potential adverse outcome in comparison to MPT. ${ }^{5}$ The 
current findings provide preliminary evidence towards a better understanding of the effects of postural drainage chest physiotherapy on GOR, arousal, and cardiorespiratory function. We speculate that the $30^{\circ}$ head-down position results in an increase in gastric fundal pressure, a decrease in gastric "reservoir capacity" and more dependent positioning of the gastro-oesophageal junction which may favour reflux. Our findings reinforce the recent statement by Orenstein ${ }^{6}$ cautioning against the routine use of SPT in asymptomatic infants with CF, particularly if manifestations of gastrooesophageal reflux disease are present. Further prospective studies examining the long term pulmonary outcome in relation to chest physiotherapy in infants with CF are needed.

\section{ACKNOWLEDGEMENTS}

The authors thank Ms Dianne Simpson, RN, Department of Gastroenterology and Clinical Nutrition, for performing the oesophageal $\mathrm{pH}$ monitoring studies. The authors also gratefully acknowledge Associate Professor Colin F Robertson, Associate Professor Phillip J Robinson, Associate Professor Susan Sawyer, and Ms Judith Glazner, Department of Respiratory Medicine, Ms Anne McCoy, Department of Physiotherapy, and Professor Joan McMeeken, School of Physiotherapy (University of Melbourne), for their encouragement, support, and assistance with the study.

\section{Authors' affiliations}

B M Button, School of Physiotherapy, The University of Melbourne, Parkville, Victoria 3052, Australia

R G Heine, A G Catto-Smith, Department of Gastroenterology and Clinical Nutrition, Royal Children's Hospital, Melbourne, Australia P D Phelan, A Olinsky, Department of Respiratory Medicine, Royal Children's Hospital, Melbourne, Australia

\section{REFERENCES}

1 Feigelson J, Sauvegrain J. Reflux oesophagien dans le mucoviscidose. Nouv Presse Med 1975;4:2729-30.

2 Heine RG, Button BM, Olinsky A, et al. Gastro-oesophageal reflux in infants under 6 months with cystic fibrosis. Arch Dis Child 1998;78:44-8.

3 Button BM, Heine RG, Catto-Smith AG, et al. Postural drainage and gastrooesophageal reflux in infants with cystic fibrosis. Arch Dis Child 1997;76:148-50.

4 Button BM, Heine RG, Catto-Smith AG, et al. Postural drainage in cystic fibrosis: is there a link with gastro-oesophageal reflux? J Paediatr Child Health 1998; 34:330-4.

5 Button BM, Heine RG, Catto-Smith AG, et al. Chest physiotherapy in infants with cystic fibrosis: to tip or not? A five-year study. Pediatr Pulmonol 2003;35:208-13.

6 Orenstein DM. Heads up! clear those airways! Pediatr Pulmonol 2003;35:160-1.
7 Kawahara H, Dent J, Davidson G. Mechanisms responsible for gastroesophageal reflux in children. Gastroenterology 1997;113:399-408.

8 Cucchiara S, Santamaria F, Andreotti MR, et al. Mechanisms of gastrooesophageal reflux in cystic fibrosis. Arch Dis Child 1991;66:617-22.

9 Orenstein SR. Effect of nonnutritive sucking on infant gastroesophageal reflux. Pediatr Res 1988;24:38-40

10 Vandenplas $Y$, De Wolf $D$, Deneyer $M$, et al. Incidence of gastroesophageal reflux in sleep, awake, fasted, and postcibal periods in asymptomatic and symptomatic infants. J Pediatr Gastroenterol Nutr 1988;7:177-80.

11 Vandenplas $Y$, Sacre-Smits L. Seventeen-hour continuous esophageal pH monitoring in the newborn: evaluation of the influence of position in asymptomatic and symptomatic babies. J Pediatr Gastroenterol Nutr 1985;4:356-61.

12 Strobel CT, Byrne WJ, Ament ME, et al. Correlation of esophageal lengths in children with height: application to the Tuttle test without prior esophageal manometry. J Pediatr 1979;94:81-4.

13 Orenstein SR, Whitington PF, Orenstein DM. The infant seat as treatment for gastroesophageal reflux. N Engl J Med 1983;309:760-3.

14 Orenstein SR. Prone positioning in infant gastroesophageal reflux: is elevation of the head worth the trouble? J Pediatr 1990;117:184-7.

15 Tobin JM, McCloud P, Cameron DJ. Posture and gastro-oesophageal reflux: a case for left lateral positioning. Arch Dis Child 1997;76:254-8.

16 Meyers WF, Herbst JJ. Effectiveness of positioning therapy for gastroesophageal reflux. Pediatrics 1982;69:768-72.

17 Orenstein SR, Shalaby TM, Cohn JF. Reflux symptoms in 100 normal infants: diagnostic validity of the infant gastroesophageal reflux questionnaire. Clin Pediatr 1996;35:607-14.

18 Orenstein SR. Crying does not exacerbate gastroesophageal reflux in infants. J Pediatr Gastroenterol Nutr 1992;14:34-7.

19 Snel A, Barnett CP, Cresp TL, et al. Behavior and gastroesophageal reflux in the premature neonate. J Pediatr Gastroenterol Nutr 2000;30:18-21.

20 Feranchak AP, Orenstein SR, Cohn JF. Behaviors associated with onset of gastroesophageal reflux episodes in infants. Prospective study using splitscreen video and $\mathrm{pH}$ probe. Clin Pediatr 1994;33:654-62.

21 Giles DR, Wagener JS, Accurso FJ, et al. Short-term effects of postural drainage with clapping vs autogenic drainage on oxygen saturation and sputum recovery in patients with cystic fibrosis. Chest 1995;108:952-4.

22 Falk M, Kelstrup M, Andersen JB, et al. Improving the ketchup bottle method with positive expiratory pressure, PEP, in cystic fibrosis. Eur $J$ Respir Dis 1984;65:423-32.

23 McDonnell T, McNicholas WT, FitzGerald MX. Hypoxaemia during chest physiotherapy in patients with cystic fibrosis. Irish J Med Sci 1986; 155:345-8.

24 Pinelli J, Symington A. Non-nutritive sucking for promoting physiologic stability and nutrition in preterm infants. (Update in Cochrane Database of Systemic Reviews 2001;(3):CD001071; PMID: 11686975). Cochrane Database of Systematic Reviews 2000;(2):CD001071.

25 Shiao SY, Chang YJ, Lannon $\mathrm{H}$, et al. Meta-analysis of the effects of nonnutritive sucking on heart rate and peripheral oxygenation: research from the past 30 years. Issues in Comprehensive Pediatric Nursing 1997;20:11-24.

26 DiPietro JA, Cusson RM, Caughy MO, et al. Behavioral and physiologic effects of nonnutritive sucking during gavage feeding in preterm infants. Pediatr Res 1994;36:207-14.

27 Treloar DM. The effect of nonnutritive sucking on oxygenation in healthy, crying full-term infants. Appl Nurs Res 1994;7:52-8.

28 Paludetto R, Robertson SS, Hack $M$, et al. Transcutaneous oxygen tension during nonnutritive sucking in preterm infants. Pediatrics 1984;74:539-42. 